\title{
Pediatric tricuspid valve replacement with a transcatheter aortic valve (SAPIEN 3)
}

\author{
Afksendiyos Kalangos, MD, ${ }^{\mathrm{a}}$ Nataliia Shatelen, MD, ${ }^{\mathrm{b}}$ Panagiotis Sfyridis, MD, ${ }^{\mathrm{a}}$ Evangelia Dalarizou, MD, ${ }^{\mathrm{a}}$ \\ and Pipina Bonou, MD, ${ }^{\mathrm{c}}$ Athens, Greece, and Kiev, Ukraine
}

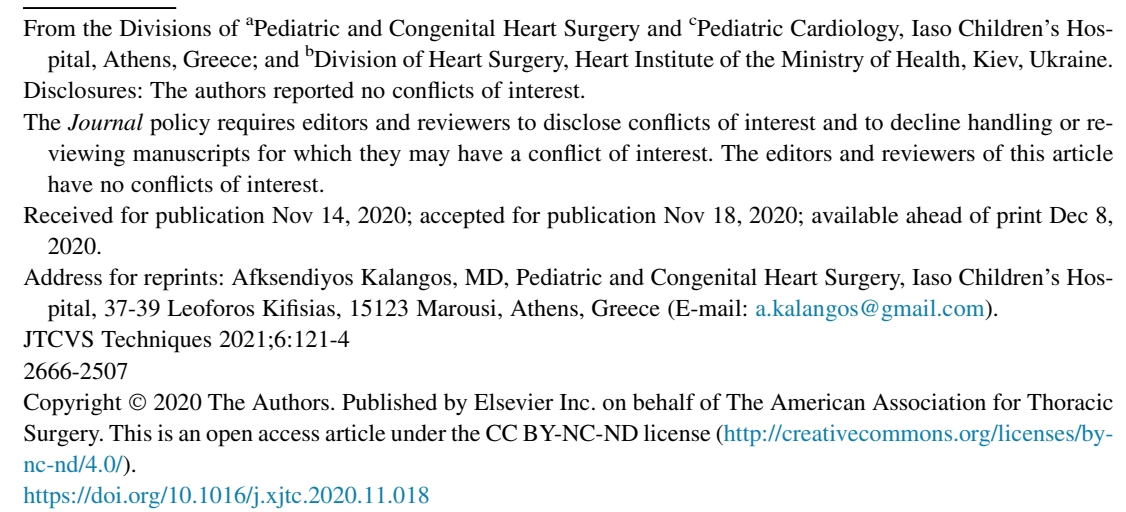

Video clip is available online.

Tricuspid regurgitation (TR) due to dysplasia in children may sometimes lead to gradual right heart failure after remaining asymptomatic during the first years of life. For this reason, tricuspid valve (TV) repair or replacement procedures become mandatory at the stage of symptomatic right heart failure, even when the diameter of the tricuspid orifice remains hypoplastic. We report a novel off-label use of the transcatheter aortic valve SAPIEN 3 (Edwards Lifesciences LLC, Irvine, Calif) in tricuspid position through a small right thoracotomy in a child. Informed consent was received for our patient.

The patient was an 8-year-old boy with a history of transposition of the great arteries associated with subpulmonary ventricular septal defect (VSD), moderate tricuspid annular hypoplasia ( $Z$ value: -3 ), straddling of the tricuspid anterior chords inserting into the left ventricular outflow tract, and coarctation of the aorta who had undergone several previous interventions: Rashkind procedure immediately after birth, coarctation repair at the age of 19 days, pulmonary banding with simultaneous atrial septal defect enlargement at the age of 34 days, and pulmonary rebanding 20 days later. Pulmonary banding was chosen by the former surgical team, who preferred to delay the arterial switch and VSD closure because of the straddling of the tricuspid anterior chords inserting into left ventricular outflow tract.

At the age of 6 months, the patient underwent arterial switch with VSD closure, pulmonary debanding combined

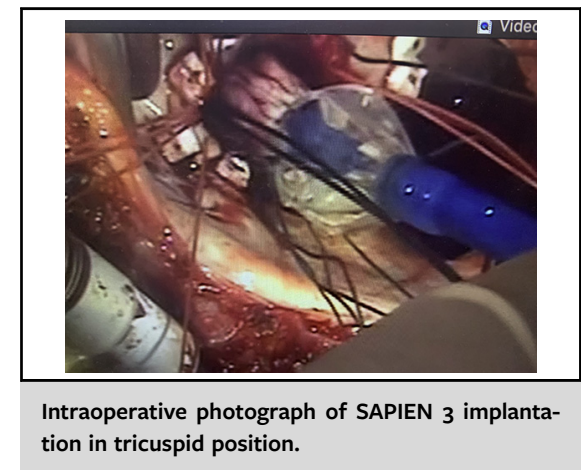

\section{CENTRAL MESSAGE \\ Transcatheter valves have started becoming an attractive option for atrioventricular valve replacement in children. We report the use of a SAPIEN 3 valve to replace the tricuspid valve in a child.}

See Commentaries on pages 125 and 126.

with patch enlargement of the pulmonary trunk, and epicardial dual-chamber pace maker implantation. After this surgery, the child, who had mild-to-moderate tricuspid stenosis since his birth due to annular hypoplasia, began developing progressive TR. At the age of 4 years, he was referred to our institution for further care, with severe TR and dilated right heart chambers. On transthoracic echocardiography (TTE), the anterior and posterior leaflets of TV were dysplastic, as characterized by thickened leaflet tissue and short primary chordae tendineae resulting in a significant coaptation gap during systole between leaflets. The right ventricular end-diastolic volume index was estimated as $146 \mathrm{~mL} /$ $\mathrm{m}^{2}$ and the right ventricular ejection fraction as $46 \%$.

The patient underwent TV repair by enlarging the anterior and posterior leaflets with an ellipsoid bovine pericardial patch. The discharge TTE showed mild residual TR and moderate tricuspid stenosis (mean transtricuspid gradient: $7 \mathrm{~mm} \mathrm{Hg}$ ). Four years after this surgery, the child developed ascites and progressive protein-losing enteropathy (PLE). On TTE, the degree of TR was severe, the 
stenotic component remaining stable at mild-to-moderate degree with a mean gradient of $5 \mathrm{~mm} \mathrm{Hg}$. Tricuspid annulus diameter was estimated as $17 \mathrm{~mm}$ (Video 1). During the surgery, cardiopulmonary bypass was initiated by directly cannulating the abdominal aorta and vena cava inferior through a right retroperitoneal approach because of the obstructed bilateral femoro-iliac arteries and veins, as previously described. ${ }^{1}$ The right atrium was then exposed by separating the pericardial adhesions through a small right anterior thoracotomy, which was opted for avoidance of a new resternotomy. The vena cava superior was cannulated through the right thoracotomy. On beating heart, TV was exposed through a right atriotomy, the thickened anterior and posterior leaflets, previously enlarged by a bovine pericardial patch, were excised and the orifice was measured by Hegar dilators. A 17-mm dilator was advanced with friction through the TV orifice. Then, 3-0 pledgeted coated braided polyester stitches were placed along the circumference of the tricuspid annulus. As the predicted diameter of the tricuspid orifice was $23 \mathrm{~mm}$, a SAPIEN 3 valve size 26 was chosen to preserve the potential for subsequent percutaneous balloon expansion as the child grows. The valve was crimped on the delivery system containing a balloon catheter without any modification or cut in the stenting structure of the valve. It was then deployed under direct vision by gradually inflating the balloon at $23 \mathrm{~mm}$ so that the basal skirt of the valve remained at the supra-annular level (Figure 1). This deployment was performed under the control of transesophageal echocardiography (TEE) on beating heart to avoid any significant compression of the aortic annulus and root or obstruction in the right ventricular outflow tract (RVOT). The 3/0 stitches already placed in the tricuspid annulus were then passed through

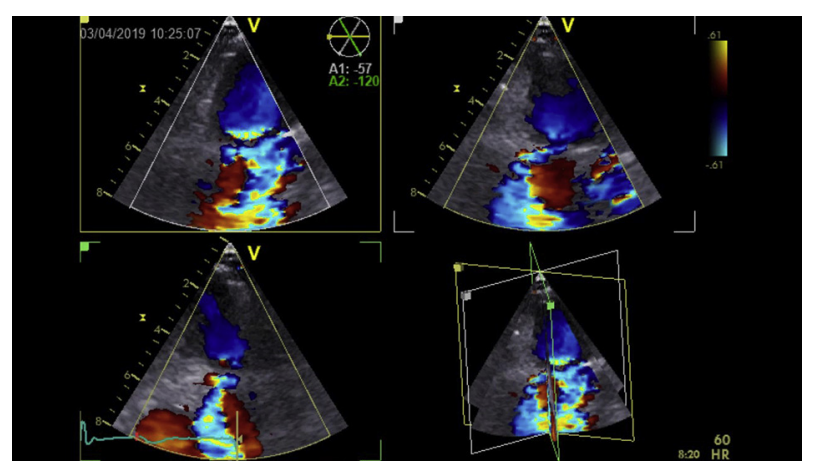

VIDEO 1. Preoperative transesophageal echocardiography. We see the fixed annulus of the tricuspid valve due to fibrosis from previous correction and the to-and-fro flow across the valve (mixed valvular disease). The regurgitant component was the main hemodynamic reason of the child's protein losing enteropathy. Video available at: https://www.jtcvs.org/article/ S2666-2507(20)30704-5/fulltext.

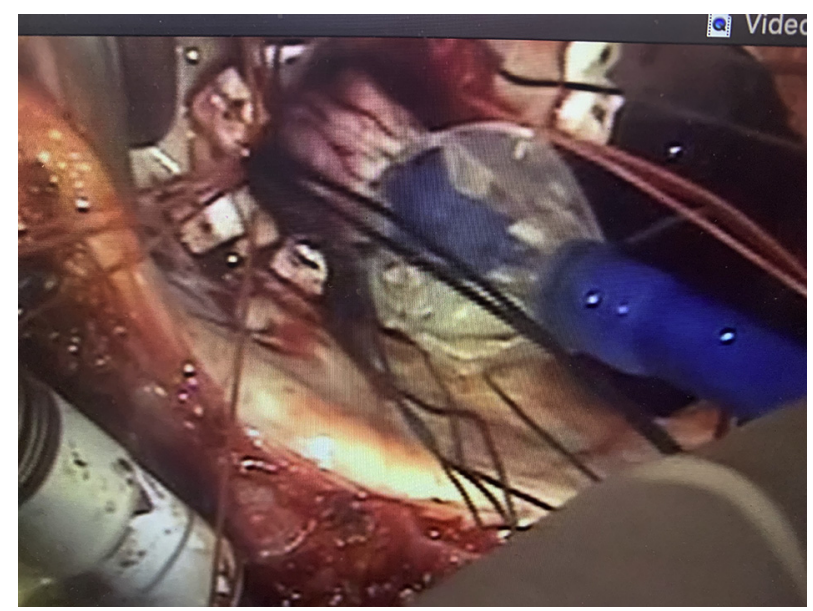

FIGURE 1. Deployment of the size-26 SAPIEN 3 valve under direct vision by gradually inflating the balloon at $23 \mathrm{~mm}$ so that the basal skirt of the valve remained at the supra-annular level.

the Dacron-reinforced basal skirt of the valve and tied on the internal side of the skirt to prevent migration (Video 2).

The patient was weaned from cardiopulmonary bypass without any hemodynamic problem under TEE control, which showed an adequate function of the SAPIEN 3 valve with no residual insufficiency and a mean transtricuspid gradient of $3 \mathrm{~mm} \mathrm{Hg}$. There was no aortic annular compression or RVOT obstruction. The postoperative course of this young patient was spectacular, with immediate resolution of ascites and PLE within 12 days of postoperative hospital stay. During his in-hospital period, the patient received 1 dose of human albumin $2 \mathrm{~g} / \mathrm{kg}$ and did not require any subsequent replacement therapy for PLE, until date. He was discharged home with lisinopril $2.5 \mathrm{mg} /$ day and diuretics (aldactone and furosemide), the doses of which were gradually reduced over 3 months postoperatively to a low prophylactic level less than $1 \mathrm{mg} / \mathrm{kg} /$ day. The patient is currently under this triple medication and preserves a

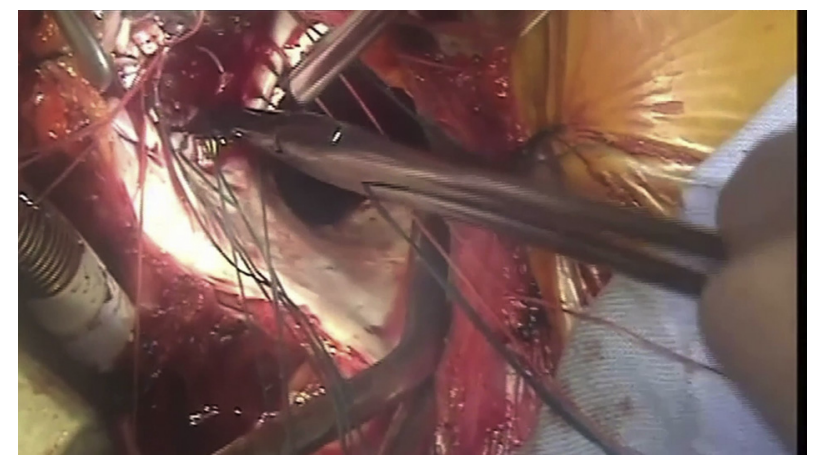

VIDEO 2. The video illustrates the surgical technique of the SAPIEN 3 valve implantation. Video available at: https://www.jtcvs.org/article/ S2666-2507(20)30704-5/fulltext. 


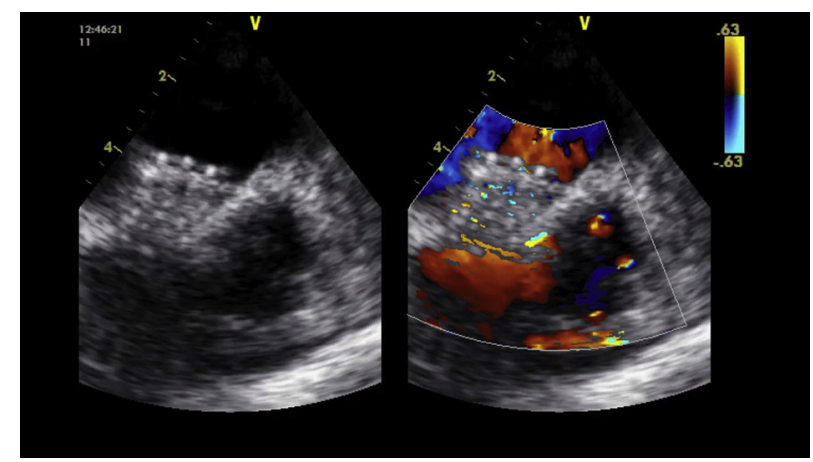

VIDEO3. Transesophageal echocardiography at postoperative 16 months' follow-up. The SAPIEN 3 valve located in tricuspid position without obstructing the flow of the right ventricular outflow tract or compressing the left ventricular outflow tract. The function of SAPIEN 3 was normal, with no stenosis or regurgitation. Mild turbulence is noted in the inflow, inducing a mean transvalvular gradient of $3 \mathrm{~mm} \mathrm{Hg}$. Video available at: https://www.jtcvs.org/article/S2666-2507(20)30704-5/fulltext.

well-balanced condition despite a right ventricular ejection fraction fluctuating between $40 \%$ and $46 \%$. Although it is well known that the SAPIEN 3 valve in atrioventricular position is prone to leaflet thrombosis, postoperative antiplatelet or anticoagulation regimen was avoided in this patient because of his severe liver dysfunction. At postoperative follow-up of 16 months, the patient's TTE findings remained unchanged (Video 3), therefore precluding the indication for dilatation of the SAPIEN 3 valve, which might be considered later based on the evolution of clinical and echocardiographic findings.

\section{DISCUSSION}

TV dysplasia is a rare congenital valve disease that may be the origin of progressive tricuspid dysfunction in infants and young children. Despite the high mortality and morbidity rates, atrioventricular valve replacement in children is unavoidable when repair is unfeasible. The lack of currently available traditional prosthetic valves for children with annulus size $<15 \mathrm{~mm}$ encouraged surgeons to look for alternative solutions in the development of recent transcatheter valves technology. ${ }^{2,3}$ Favorable design characteristics of these valves amenable to subsequent catheter-based valve expansion decrease the risk of early reoperation by accommodating the valve size of the children to their somatic growth. Surgically implanted Melody valve in tricuspid position of a 5-month-old child was previously reported with a favorable initial outcome. ${ }^{4}$

In our case, the diameter of the tricuspid orifice estimated as $17 \mathrm{~mm}$ on TTE was confirmed intraoperatively by the 17mm Hegar dilator, which was advanced with friction through the tricuspid orifice despite the resection of the anterior and posterior tricuspid leaflets. The option for tricuspid replacement with a $17-\mathrm{mm}$ mechanical valve was precluded due to noncompliance of our patient to anticoagulants related to his delayed mental development. The main reason in favor of using a transcatheter balloon expandable valve was the intention to upsize the hypoplastic tricuspid orifice from $17 \mathrm{~mm}$ to $23 \mathrm{~mm}$, which corresponds to the predicted value based on the child's body surface area. For this reason, the balloon was gradually expanded to $23 \mathrm{~mm}$ under TEE guidance during beating heart surgery to avoid any deformation or compression of the aortic annulus or any obstruction in RVOT caused by the valve stents. This fact constitutes the uniqueness of our case compared with that previously reported with the Melody valve. ${ }^{4}$ The permanent pacemaker implanted after the previous arterial switch and VSD closure precluded us from evaluating the risk of potential injury on the conduction system, which might have occurred due to overstretching the tricuspid annular diameter from 17 to $23 \mathrm{~mm}$ during the gradual balloon expansion with the SAPIEN 3 valve. The alternative delivery of a Melody valve through the surgically exposed inferior vena cava or jugular vein was considered risky due to the potential risk of transcatheter valve migration and the mandatory use of a 22-French sheath for transvenous placement, which was considered too large for this pediatric patient with a small body surface area of $0.70 \mathrm{~m}^{2}$. For this reason, the surgical deployment of a transcatheter valve was preferred. As the biggest size of the Melody valve is $22 \mathrm{~mm}$, it would not have been suitable in our case to overexpand the balloon to $23 \mathrm{~mm}$ during deployment and consider subsequent catheter-based reexpansion up to $26 \mathrm{~mm}$ at a later stage. The height of Melody valve's stents decreases from 26 to $21 \mathrm{~mm}$ as the valve size increases from 18 to $22 \mathrm{~mm}$. In fact, as the height of the size-26 SAPIEN 3 valve $(20 \mathrm{~mm})$ is very close to that of the size-22 Melody valve $(21 \mathrm{~mm})$, this parameter did not play any role in favor of our choice of the SAPIEN 3 valve. Compared with the Melody valve, for which the biggest size is 22 , the SAPIEN valve sizes 26 and 29 can obviously offer wider opportunities of use for the replacement of both atrioventricular valves, especially in bigger children. The circumferential fixation of a bovine pericardial strip around the Melody valve's cage may sometimes be the underlying cause of early or late appearance of paravalvular leaks from the fixation stitches. The need for this pericardial strip can be considered another technical disadvantage compared with SAPIEN 3, which is anchored to the native annulus using simple pledget-reinforced stitches. The favorable outcome during the initial 16 months of follow-up in our case may encourage surgeons to consider the use of transcatheter valves as an alternative option to conventional small-size surgical valvular prostheses. Pediatric cases with hypoplastic atrioventricular valve annulus can especially benefit from the potential accommodation of their hypoplastic annulus to the predicted value at the time of initial 
transcatheter valve implantation. There is no published long-term experience regarding late dilatation of transcatheter valves in any valve position in children who have outgrown the initial valve dimension. We may speculate that the safety and efficacy of transcatheter valve redilatation would be similar to those of stents implanted for aortic coarctation or pulmonary branch stenoses in children, ${ }^{5}$ despite the lack of studies determining whether these valves would remain competent or their dilatation would result in tearing of leaflets or other components. However, these valves may potentially offer the opportunity of serial balloon dilatations to maintain an adequate effective orifice over time in growing children.

\section{References}

1. Sfyridis PG, Mylonas KS, Kalangos A. Abdominal vessel cannulation prior to resternotomy in complex congenital heart surgery. Ann Thorac Surg. 2020;109: e219-21.

2. Fernandez-Doblas J, Perez-Andreu J, Betrian P, Abella RF. Pediatric tricuspid valve replacement with transcatheter bioprosthetic valve: an alternative option in high-risk patients. Semin Thorac Cardiovasc Surg. 2020; 32:1021-3.

3. Pluchinotta FR, Piekarski BL, Milani V, Kretschmar O, Burch PT, Hakami L, et al. Surgical atrioventricular valve replacement with Melody valve in infants and children. Circ Cardiovasc Interv. 2018;11:e007145.

4. Hermsen JL, Permut LC, McQuinn TC, Lones TK, Chen JM, McMullan DM. Tricuspid valve replacement with a Melody stented bovine jugular vein conduit. Ann Thorac Surg. 2014;98:1826-7.

5. Duke C, Rosenthal E, Qureshi SA. The efficacy and safety of stent redilatation in congenital heart disease. Heart. 2003;89:905-12. 